\title{
Feasibility of Interlaminar Lumbar Discectomy through Percutaneous Transforaminal Endoscopy and Blunt Perforation of the Ligamentum Flavum
}

\author{
Baoshan HU, Gang RUI, Naikun SUN, Jinyi FENG, Shengrong LIN \\ The First Affiliated Hospital of Xiamen University, Department of Spine Surgery, Xiamen, Fujian, China
}

\section{ABSTRACT}

AIM: To determine the efficacy, safety, and clinical value of a novel surgical procedure involving the blunt perforation of the ligamentum flavum (LF) during endoscopic interlaminar lumbar discectomy.

MATERIAL and METHODS: This was a prospective study of 50 patients (25 males, 17-51 years of age) undergoing lumbar discectomy for single segment L4/L5 or L5/S1 disc herniation. They were grouped into the control (cutting of the LF; $n=22$ ) and test (blunt perforation; $n=28$ ) groups. Intraoperative injury to the LF was evaluated by electrophysiological monitoring. The time required for perforation, total surgical time, and proportion of epidural sac and nerve root injury were assessed.

RESULTS: Among the enrolled patients, $10 \%$ showed herniation of the L4/5 segment and $90 \%$ of the L5/S1 segment. The success rate for the perforation of LF was $93 \%$. The intraoperative observation showed mild self-closing injury to the LF tissue. The test group showed shorter overall surgical time ( 43 vs. 56 minutes) and shorter duration to go through the LF ( 1 vs. 13 minutes, $p<0.001)$. No dural sac or nerve root injury resulting from blunt perforation of the LF was observed.

CONCLUSION: Compared to cutting, blunt perforation of the ligamentum flavum could reduce surgical time and injury to ligamentum flavum and surrounding tissues. Thus, it could be a safe and efficient surgical technique for patients undergoing interlaminar lumbar discectomy.

KEYWORDS: Interlaminar, Ligamentum flavum, Lumbar disc herniation, Transforaminal endoscopic discectomy

\section{INTRODUCTION}

$\mathrm{I}$ $n$ recent years, success has been achieved in the treatment of lumbar disc herniation owing, in part, to the Luse of minimally-invasive percutaneous transforaminal endoscopic discectomy $(13,16)$. The success of percutaneous endoscopic techniques is largely due to low tissue trauma and better postoperative recovery.

One of the key procedures for discectomy via the posterior, transforaminal, and percutaneous endoscopic approaches requires breaking through the ligamentum flavum (LF) to expose the injured disc. Although significant improvements have been made in controlling the intraoperative injury to the dural sac and nerve root, the best approach to break through the LF safely remains a pivotal issue (15). An early technique was the puncture and gradual dilation of the LF under fluoroscopy, but this method was largely abandoned due to the high incidence of subsequent dural sac and nerve root injuries. Current techniques include the cutting, burning, and splitting of the ligament under direct vision; they are considered safe, but are also time-consuming $(14,16)$. New approaches for faster and safe LF perforation are needed to improve the surgical outcomes. 
Therefore, this study aimed to compare the efficacy and safety of a new technique, i.e. the blunt perforation of the LF, with the conventional method of cutting the LF, and to assess its clinical value in terms of surgical time, injury to the LF, and rate of perioperative dural sac and nerve root injuries.

\section{MATERIAL and METHODS}

\section{Study Design and Patients}

A total of 50 patients diagnosed with lumbar disc herniation at the Orthopedic Department of The First Affiliated Hospital of Xiamen University between March 2014 and March 2015 were prospectively and consecutively enrolled.

The patients diagnosed with a single segment disc herniation were eligible if they either: 1) had symptoms for at least six months prior to surgery for which conservative treatment had failed; 2) had severe symptoms interfering with movement or sleep; 3) had a prolonged disease history affecting daily living; 4) had continuously deteriorating neurological symptoms; or 5) had atypical symptoms but a huge protrusion confirmed by computed tomography (CT) or magnetic resonance imaging (MRI). Patients with nucleus pulposus extrusion and a highdegree of disassociated replacement were excluded. Patients with a laminar space smaller than $0.8 \mathrm{~cm}$ in diameter (Figure 1A) were also excluded because the trocar was unable to pass through the space. Patients with evidence of LF calcification (Figure 1B) or abnormal LF thickening that was hard to distinguish from the dural sac were also excluded.

This study was approved by the ethical committee of The First Affiliated Hospital of Xiamen University. Written informed consent was provided by all patients.

\section{Grouping}

Among the 50 enrolled patients, the first 22 patients were operated using the cutting method to break through the LF; these patients were considered as the control group. The latter 28 patients were operated using blunt perforation to break through the LF to perform endoscopic percutaneous transforaminal discectomy; they were considered as the experimental group. All patients were subjected to electrophysiological monitoring during the surgery. The occurrence of LF injury was observed during the operation. The breakthrough time was recorded. The incidence of dural sac and nerve root injuries after breakthrough was assessed.

\section{Surgical Strategy for Blunt Perforation}

After epidural anesthesia, the patients were placed in the prone position with blocking floatover up to keep the abdomen vacant. Monitoring electrodes were set up at the operating nerve root to continuously monitor spontaneous electromyography (EMG) activity and changes in somatosensory evoked potentials, and to intermittently monitor changes in motor evoked potentials.

The surgical segment was positioned using a C-arm. The insertion point was selected at the medial side of the midpoint between the spinous process and facet. A $0.7-\mathrm{cm}$ incision was made through the skin and fascia. A multi-stage dualchannel positioning dilator was placed through the skin, fascia, and muscles (preferably at the intersection of the lower edge of the upper vertebral lamina and the LF) to the surface layer of the LF. The channel could enter the LF through one of two approaches, determined according to the anatomic relationship of the lumbar nerve root (Figure 2). To avoid damaging the nerve root, the running direction of the dilation channel was adapted to the preoperative under-arm or onshoulder protrusion types. For on-shoulder type protrusion, the tip of the blunt channel was pointed to the I region because the involved nerve root often shifted medially by compressing the protruded tissues. On the other hand, for under-arm type protrusion, the tip of the blunt channel was normally pointed to the II region because the involved nerve root often shifted to the lateral side by compression of the protruded tissues (Figure 2).

The positioning dilator was slowly gyrated to bluntly break the LF. Breakthrough of the LF was considered successful in
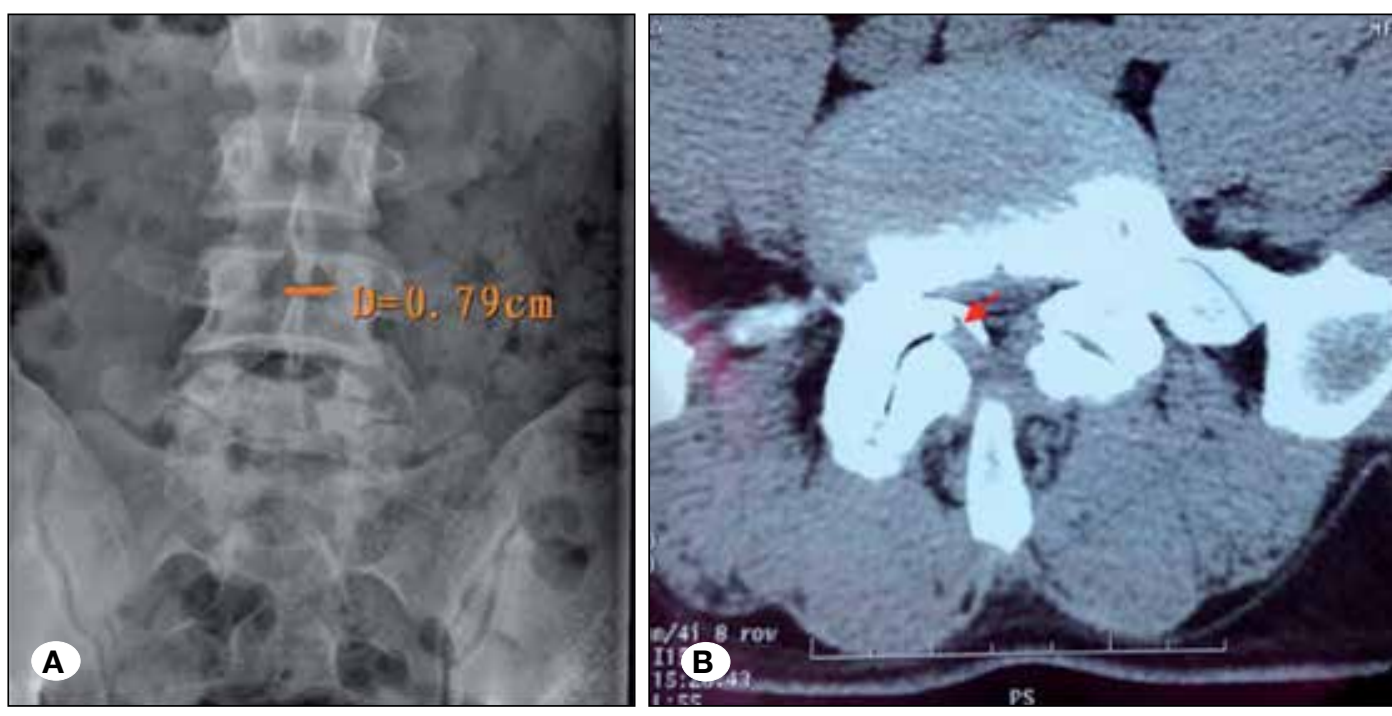

Figure 1:

A) Pre-surgical anteroposterior measurement of the laminar space by the posterior approach. B) Obvious calcification of the lumbar LF on preoperative CT scan. 
any of these conditions: 1) the epidural space below the LF demonstrated a significant loss of resistance; 2) the channel, which was prone to changes in direction before passing through the LF, was anchored in place by the surrounding tension of the LF; or 3) a further channel insertion of approximately 0.5 $\mathrm{cm}$ was achieved after loss of resistance.

After passing through the LF, a trocar was assembled and a transforaminal endoscope was inserted to observe the fat under the LF, as well as the dural sac and nerve root. If needed, the trocar was slightly retracted to identify the incision edge of the LF under endoscopy and to further confirm the penetration of the LF.

After the anatomic structures were identified, the surgery was completed by grabbing and removing the protruding nucleus pulposus of the injured intervertebral disc with nucleus pulposus forceps, bipolar ablator, endoscopic scissors, and other instruments using standard surgical techniques.

\section{Outcomes}

During surgery, all patients underwent electrophysiological monitoring in order to visualize intraoperative injury of the LF and the incidence of secondary injury to the dural sac or nerve root after penetration of the LF. All surgical segments were reevaluated with a $\mathrm{MRI}$ scan at 3 months postoperative to determine the surgical success and to evaluate the protrusion of the lumbar disc at the corresponding segment. Perisurgical

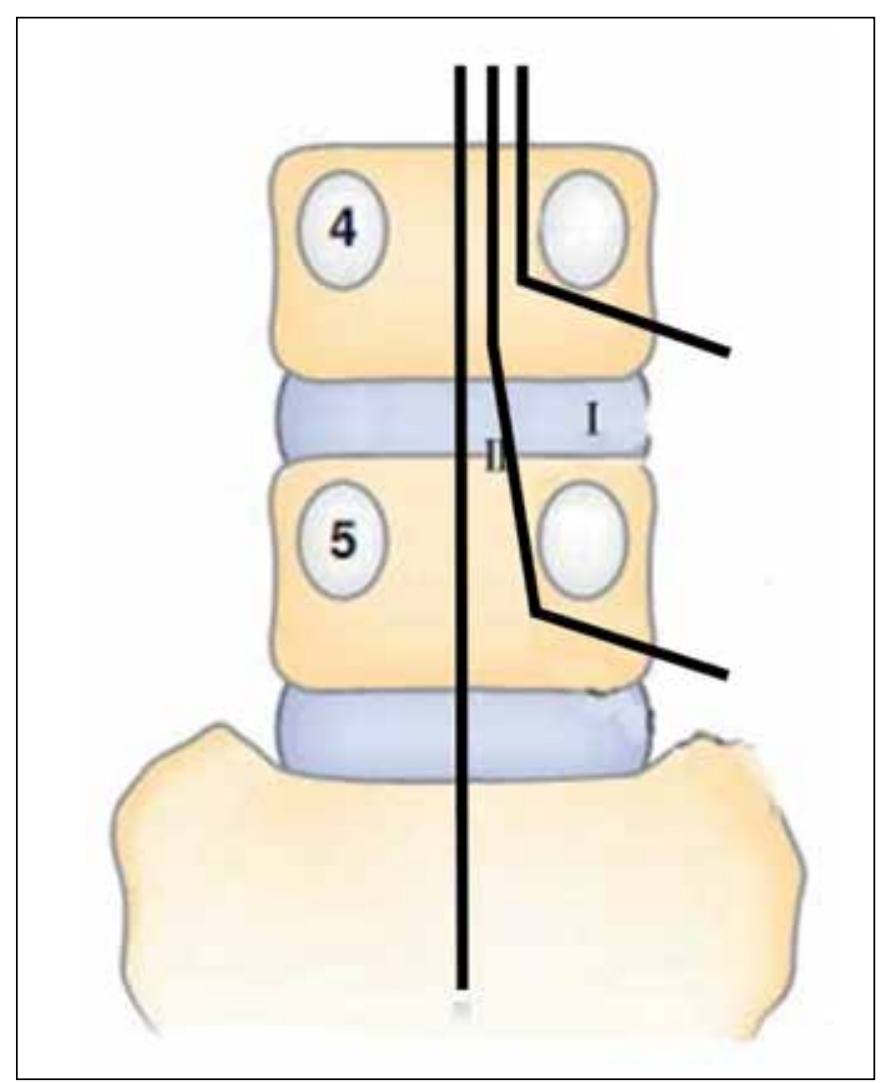

Figure 2: Running direction of the dilation channel, according to the under-arm and on-shoulder preoperative protrusion types. injury to the nerve root and dural sac, when perforating the LF, was endoscopically determined during surgery. The breakthrough time in the test group started from the gyrated breakthrough of the LF, while the breakthrough time in the control group started from the insertion of the catheter and exploration of the LF, and the end time was when the dural sac and nerve root were clearly observed after catheter entry into the spinal canal. The breakthrough time in the two groups was calculated, and the mean values were used for analysis.

Lumbar pain was assessed preoperatively using visual analogue scales (VAS) and the Macnab comprehensive score; they were re-assessed at 1,3 , and 6 postoperative months. The Macnab comprehensive assessment is a scale evaluating symptom resolution and functioning and defines patients outcomes as excellent (symptoms have completely disappeared and patients have returned to normal life and work), good (patients have mild symptoms and slightly limited activities, which do not affect their daily life and work), moderate (patients have less symptoms than they did before surgery and limited activities, which affects their daily life and work), or poor (postoperative symptoms show no improvement or worsened compared with the preoperative symptoms).

\section{Statistical Analysis}

Continuous variables were presented as mean \pm standard deviation and analyzed using the Student t test. Categorical variables were presented as frequencies and analyzed using the Fisher's exact test. VAS and the MacNab score were analyzed using the non-parametric Mann-Whitney $U$ test. SPSS 16.0 software (IBM Corp., USA) was used for statistical analysis. $\mathrm{p}<0.05$ was considered statistically significant.

\section{RESULTS}

\section{Patient Characteristics}

A total of 50 patients who underwent discectomy for single lumbar disc herniation at our hospital between March 2014 and March 2015 were prospectively enrolled. The study group included 25 males and 25 females, 17-51 years of age (mean age of 32.6 years). Most patients (45/50) had herniation of the L5/S1 segment, while the remaining five patients had herniation of the L4/L5 segment. There were no significant differences between groups for age, gender, injured segments, or preoperative VAS score (Table I). Overall, 26 of the 28 patients who underwent blunt perforation (Table I) had successful perforation of the LF, yielding a success rate of $93 \%$. Postoperative symptoms were immediately relieved in all patients, and they were discharged on the first day after surgery.

\section{Surgical Outcomes and Complications}

The surgeries were successfully performed in all patients of both groups. Intraoperative bleeding was less than $10 \mathrm{ml}$ in all patients. No abnormally reduced signals were found during intraoperative spinal monitoring, suggesting that there was no nerve root injury in all patients. Two patients who were to be operated by blunt perforation had to be switched to the classical cutting approach because of unsuccessful 
breakthrough of the LF; these two patients were excluded. In one of the patients operated by the classical approach, we observed one error in locating the injured segment. After preoperative positioning and cutting of the LF in a patient planned for L5/S1 discectomy, no nerve root compression and nucleus prolapse were observed at the L5/S1 segment, while repositioning found a prolapse at the $L 4 / 5$ segment, which was corrected without any adverse consequences. This was likely caused by sliding and displacement of the channel while replacing the instruments. Therefore, for subsequent cases, confirmation was conducted again by routine fluoroscopy after the channel was inserted.

\section{Postoperative Follow-up}

Symptoms were immediately relieved in all the patients after surgery. All patients were discharged on the first day after surgery. The patients were followed-up at 1, 3, and 6 months after surgery (Figure 3A-D).

The VAS scores for waist pain and leg pain were significantly reduced in all patients and the reduction improved over time. Pain scores were similar between the two groups (Figure 4). At six months after surgery, the Macnab assessment in the control group showed 20 excellent cases, two good cases, and no moderate case, while the test group had 23 excellent cases, two good cases, and one moderate case (Table II).

Two patients, one in each group, reported worsening lumbocrural pain on the affected side after surgery. Both patients received outpatient and close telephone follow-up. The patient who underwent discectomy using blunt perforation reported worsening pain 5 days following surgery until the pain disappeared 1 month later. The patient in the control group reported pain 7 days after surgery, which improved after 10 days, and completely disappeared at about 1 month. Both patients showed immediate and significant postoperative pain relief, while the pain was gradually aggravated after 5-7 days and was most severe at about 15 postoperative days; it was alleviated after lasting for 4-7 days, and completely disappeared at about 1 postoperative month. Both patients were successfully treated with celecoxib, a non-steroidal antiinflammatory drug.

Overall, the total surgical time ranged from 38 to 90 minutes. Compared to the control group, the use of blunt perforation was associated with reduced surgical time (43 vs. 56 minutes) and a statistically significant reduction in the time required to go through the LF (1 vs. 13 minutes, $\mathrm{p}<0.01)$.

Table I: Baseline Characteristics of the Subjects

\begin{tabular}{lccc}
\hline & Blunt perforation* (Test group) & Cutting (Control group) & p value \\
\hline Number of cases & 26 & 22 & - \\
\hline Mean age (years) \pm SD & $33.1 \pm 8.3$ & $31.9 \pm 7.6$ & 0.607 \\
\hline Gender (M:F) & $12: 14$ & $13: 9$ & 0.371 \\
\hline Surgical segment & & & 2 \\
\hline L4/L5 & 3 & 20 & 0.843 \\
\hline L5/S1 & 23 & 0.843 \\
\hline
\end{tabular}

*Successful blunt perforation.

Table II: Pain Scores at Post-Operative Follow-up

\begin{tabular}{lccc}
\hline & Blunt perforation (Test group) & Cutting (Control group) & p value \\
\hline Preoperative VAS score & $8.7 \pm 1.1$ & $8.3 \pm 1.2$ & 0.224 \\
\hline VAS score at post-operative follow-up & & & \\
\hline 1 day & $2.4 \pm 1.5$ & $2.3 \pm 1.1$ & 0.791 \\
\hline 1 month & $0.9 \pm 0.8$ & $1.4 \pm 1.0$ & 0.158 \\
\hline 3 months & $1.0 \pm 1.2$ & $1.4 \pm 0.9$ & 0.059 \\
\hline 6 months & $0.7 \pm 1.4$ & $0.4 \pm 0.5$ & 0.410 \\
\hline Macnab assessment & & & - \\
\hline Excellent & $23(88 \%)$ & $20(91 \%)$ & - \\
\hline Good & $2(8 \%)$ & $2(9 \%)$ & - \\
\hline Moderate & $1(4 \%)$ & $0 \%)$ & \\
\hline
\end{tabular}




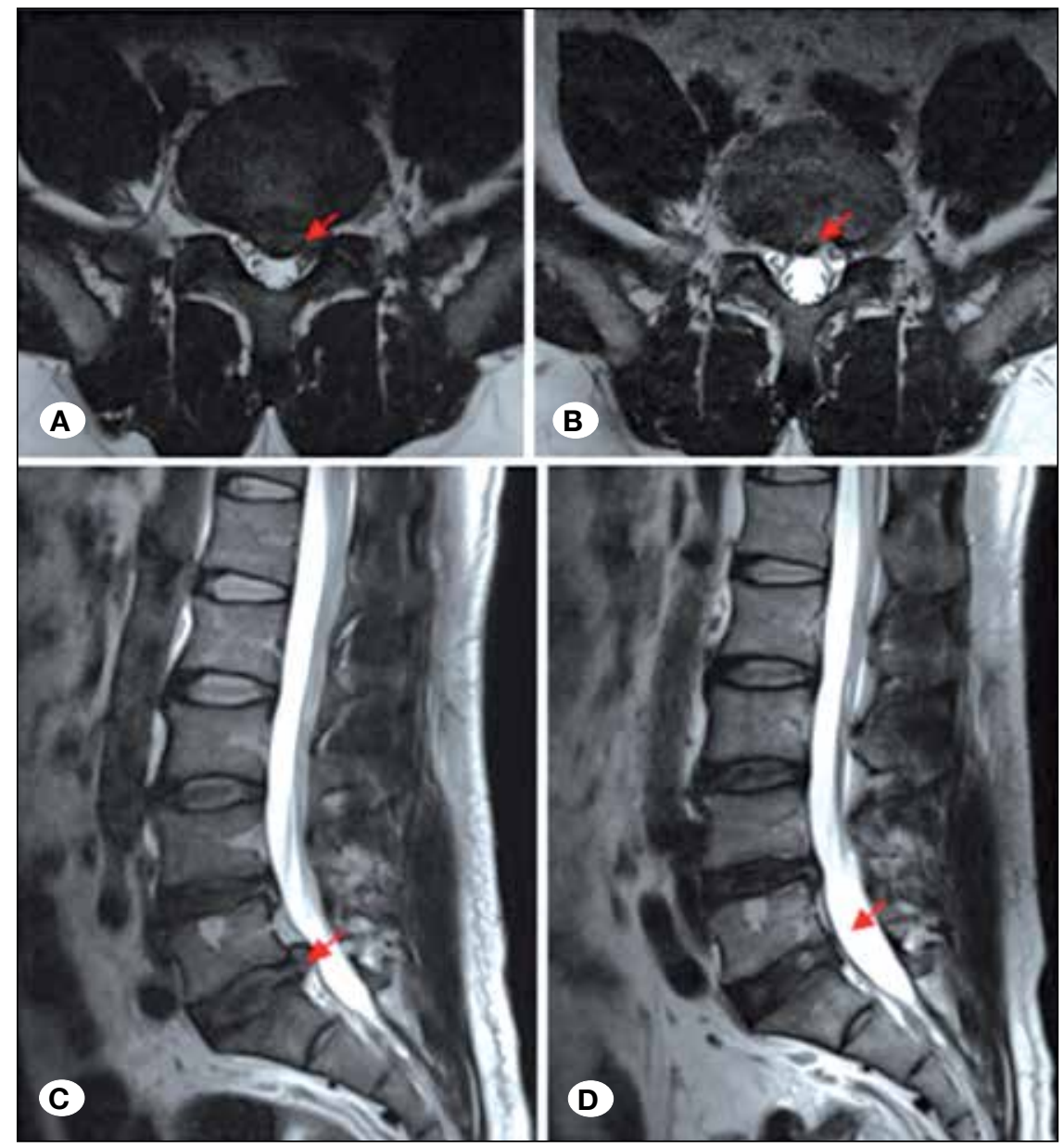

Figure 3: Cross-sectional and sagittal MRI images before surgery and at 3 months after surgery. A) Preoperative MRI (horizontal position) showing that the left side of L5/S1 was extruded and the dural sac of the nerve root was compressed. B) Three months later, MRI showing that the dural sac compression was alleviated. C) Before surgery, sagittal MRI showing that the L5/S1 protrusion compressed the dural sac. D) Three months later, sagittal MRI showing that the prominent disc had disappeared, and the dural sac compression was alleviated.

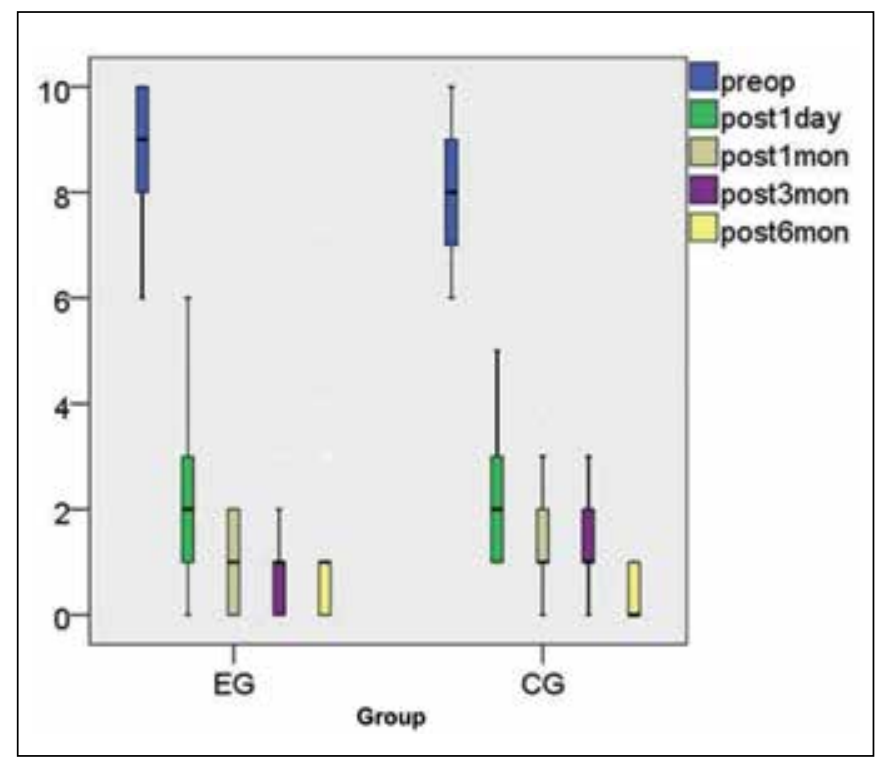

Figure 4: Pre-surgery and follow-up results. The preoperative results are in blue, results for 1 day after surgery are in green, results for 1 month after surgery are in blue, results for 3 months after surgery are in purple, and results for 6 months after surgery are in yellow. EG: test group, CG: control group.
No dural sac or nerve root injury resulting from blunt perforation of the LF was observed. A single injury to the nerve root epineurium was observed; it did not affect the endoneurium and it occurred after the LF was broken through and the adhesive nerve root was stripped; it was unrelated to the technique of blunt perforation.

\section{DISCUSSION}

There are many different surgical techniques for lumbar discectomy and, over the last decades, a variety of minimally invasive methods have gradually emerged $(5,14,16)$. Endoscopic percutaneous transforaminal discectomy has been considered an important breakthrough to provide a successful minimally invasive approach $(2,5,9,13,16)$, for which the posterior interlaminar approach is currently one of the most common $(3,6)$. Breaking through the LF is the cornerstone of discectomy, but this requires a highly skilled technique, has a high risk of damaging the nerve root and dural sac (17), and is associated with longer surgical time. Puncture into the intervertebral disc, through a posterior approach, was previously a common method, but it was later deemed unsafe and was replaced by cutting or burning open the ligament under direct or endoscopic vision $(8,18)$. 
In this study, we demonstrated that blunt perforation of the LF through the working channel constitutes a new technique that appears to be effective and safe in patients undergoing lumbar discectomy. This method can be divided into two different approaches. First, it is possible to directly poke open the LF through the working channel $(1,4,7,12)$. This method is safe and operated under endoscopy, but the channel is thick and its oval end may cause larger injury to the LF. Another approach, which was demonstrated in this paper, requires the insertion of a dual-channel positioning dilator to the LF via the skin incision, which is directly gyrated into the LF before setting up a working channel. Since the positioning dilator has a blunt tip, it can spontaneously dilate when it is passed through the $\mathrm{LF}$, leading to less damage. Following insertion into the spinal canal, the dural sac and nerve root can be identified under clear endoscopic vision. Our results suggest that compared to the standard cutting techniques, blunt perforation requires less surgical time to break through the LF. The surgical time was only 1.2 minute for patients in the perforation group, compared to an average of 13.1 minutes in the control group.

The use of a multi-stage dual-channel dilator is unlikely to damage the dural sac and nerve root when it is gyrated through the LF because of its blunt tip and the higher elasticity of the dural sac and nerve root (Figure 5). In this study, no dural sac or nerve root injury resulting from blunt perforation of the LF was observed. In addition, because it does not require cutting or burning open the LF tissue, instead breaking through the fibrous connections by passing through the LF in a planar manner, our technique minimizes damage and trauma to the LF by applying a gyrated external force. In all patients, the LF appeared completely closed after retracting the channel, with almost no traces of injury (Figure 6). Other studies suggest that methods for preserving the LF are helpful, with improved clinical outcomes, which may have resulted from the prevention of fibrosis-related complications $(10,11)$. Third, breaking through the LF does not require endoscopy and it is therefore unnecessary to strip or ablate the LF and almost no damage occurs to the connective tissues outside of the LF. Compared with the cutting method, blunt perforation only requires the correct identification of the affected segment.

To ensure the success rate of this technique, a position of the post-lumbar protrusion must be maintained to keep the laminar space open and, more importantly, to maintain traction tension on the LF. In addition, patients with preoperative CT or MRI evidence of severe lumbar degeneration, significant hyperplasia, or poor elasticity of the LF may have a higher risk of dural sac and nerve root injury and should not be considered adequate candidates for this technique. For lessexperienced surgeons, it is also preferable to administer long-lasting epidural anesthesia in order to maintain partial pain sensation in the nerve root and enable an intraoperative wakefulness state of the nerve root, preventing damage to the dural sac and nerve root from aggressive surgery.

This technique has several shortcomings about which surgeons should be aware. First, it carries potential risks in patients with spinal stenosis, especially for patients with LF thickening and calcification of lumbar disc herniation. The effectiveness and safety of transforaminal endoscopic surgeries in this population have not been established and requires further study. This technique also requires a skilled surgeon with several years of experience. Most importantly, it requires a strong capacity to envision the three-dimensional conformation of local anatomic structures. Finally, our sample size is small, may be too small to highlight statistically significant differences in outcomes between the groups. Further studies are necessary to confirm the long-term clinical safety and effectiveness of this technique, as well as its clinical benefits.

\section{- CONCLUSION}

Transforaminal endoscopy is characterized by reduced trauma and intraoperative bleeding, the absence of interfering spinal stability, and quick postoperative restoration, with comparable postoperative efficacy with traditional surgical techniques. We demonstrated the effectiveness, safety, and clinical value of a new technique relying on blunt perforation of the LF.

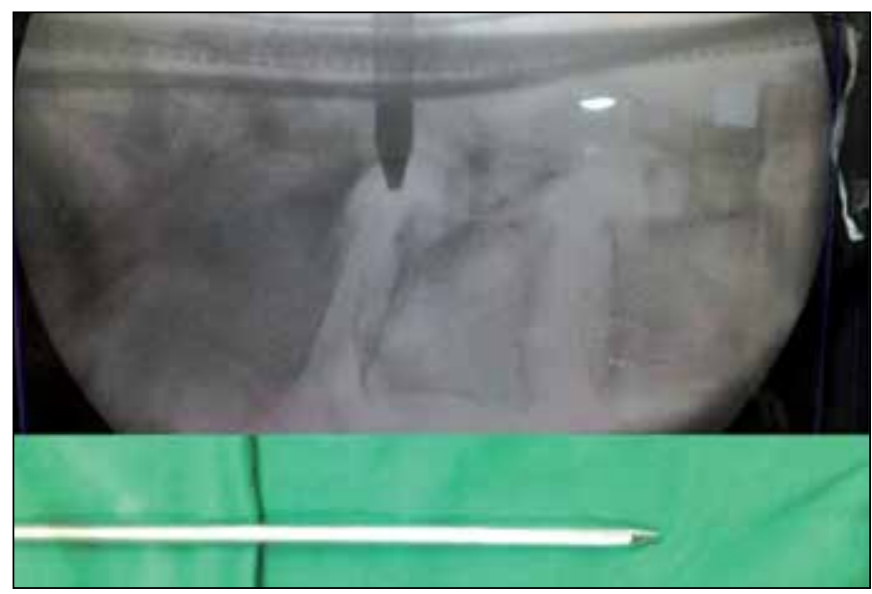

Figure 5: Blunt tip of the dilator and intraoperative imaging.

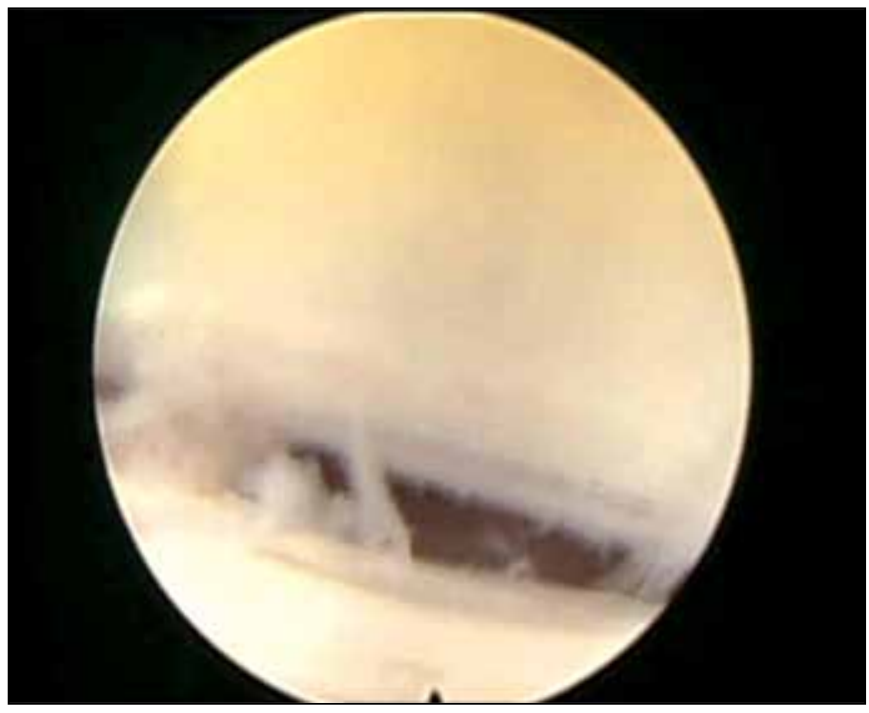

Figure 6: Fracture in the LF was closed after the surgery, showing smooth edges, longitudinal splitting of the fibers and absence of significant damage. 


\section{- ACKNOWLEDGMENT}

This work was supported by a research grant from the Foundation of Xiamen Science \& Technology Bureau (NO. 3502Z20154002).

\section{- REFERENCES}

1. Altinkaya N, Yildirim T, Demir S, Alkan O, Sarica FB: Factors associated with the thickness of the ligamentum flavum: Is ligamentum flavum thickening due to hypertrophy or buckling? Spine (Phila Pa 1976) 36: E1093-1097, 2011

2. Chen ZG, Fu Q: Percutaneous transforaminal endoscopic discectomy through different approaches for lumbar disc herniation. Zhongguo Gu Shang 25:1057-1060,2012 (In Chinese)

3. Choi G, Lee SH, Deshpande K, Choi H: Working channel endoscope in lumbar spine surgery. J Neurosurg Sci 58:7785, 2014

4. Choi G, Lee SH, Raiturker PP, Lee S, Chae YS: Percutaneous endoscopic interlaminar discectomy for intracanalicular disc herniations at L5-S1 using a rigid working channel endoscope. Neurosurgery 58: ONS 59-68; discussion ONS 59-68, 2006

5. Choi KC, Kim JS, Ryu KS, Kang BU, Ahn Y, Lee SH: Percutaneous endoscopic lumbar discectomy for L5-S1 disc herniation: Transforaminal versus interlaminar approach. Pain Physician 16:547-556, 2013

6. Jasper GP, Francisco GM, Telfeian A: Outpatient, awake, ultra-minimally invasive endoscopic treatment of lumbar disc herniations. R I Med J (2013) 97:47-49, 2014

7. Kim $\mathrm{CH}$, Chung $\mathrm{CK}$ : Endoscopic interlaminar lumbar discectomy with splitting of the ligament flavum under visual control. J Spinal Disord Tech 25:210-217, 2012

8. Kim HS, Park JY: Comparative assessment of different percutaneous endoscopic interlaminar lumbar discectomy (PEID) techniques. Pain Physician 16:359-367, 2013

9. Koga S, Sairyo K, Shibuya I, Kanamori Y, Kosugi T, Matsumoto $H$, Kitagawa Y, Sumita T, Dezawa A: Minimally invasive removal of a recurrent lumbar herniated nucleus pulposus by the small incised microendoscopic discectomy interlaminar approach. Asian J Endosc Surg 5:34-37, 2012
10. Li S, Xia H, Han C: Retrospective analysis on correlation factors of preserving the ligamentum flavum in microendoscopic discectomy. Clin Neurol Neurosurg 139:46-50, 2015

11. Li T, Han D, Liu B, Zhang X, Wang P, Qiu Y: Clinical assessment of reformed lumbar microdiscectomy. Eur J Orthop Surg Traumatol 24: 23-27, 2014

12. Li ZZ, Hou SX, Shang WL, Song KR, Zhao HL: The strategy and early clinical outcome of full-endoscopic L5/S1 discectomy through interlaminar approach. Clin Neurol Neurosurg 133: 40-45, 2015

13. Rasouli MR, Rahimi-Movaghar V, Shokraneh F, MoradiLakeh M, Chou R: Minimally invasive discectomy versus microdiscectomy/open discectomy for symptomatic lumbar disc herniation. Cochrane Database Syst Rev Cd010328, 2014

14. Ruetten S, Komp M, Merk H, Godolias G: Use of newly developed instruments and endoscopes: Full-endoscopic resection of lumbar disc herniations via the interlaminar and lateral transforaminal approach. J Neurosurg Spine 6:521530, 2007

15. Ruetten S, Meyer O, Godolias G: Endoscopic surgery of the lumbar epidural space (epiduroscopy): Results of therapeutic intervention in 93 patients. Minim Invasive Neurosurg 46:1-4, 2003

16. Sencer A, Yorukoglu AG, Akcakaya MO, Aras Y, Aydoseli A, Boyali O, Sencan F, Sabanci PA, Gomleksiz C, Imer M, Kiris T, Hepgul K, Unal OF, Izgi N, Canbolat AT: Fully endoscopic interlaminar and transforaminal lumbar discectomy: Shortterm clinical results of 163 surgically treated patients. World Neurosurg 82: 884-890, 2014

17. Takahashi $Y$, Sato T, Hyodo $H$, Kawamata T, Takahashi E, Miyatake N, Tokunaga M: Incidental durotomy during lumbar spine surgery: Risk factors and anatomic locations. Clinical article. J Neurosurg Spine 18: 165-169, 2013

18. Zhang XF, Wang Y, Xiao S: Treatment of L5/S1 disc herniation with different endoscopic approaches. Chinese Journal of Orthopaedics 3:341-345, 2010 